\title{
Empirical Modeling of Fenton Advanced Oxidation Processes for the Degradation of Linear Alkylbenzene Sulfonates
}

\author{
Ahmadi Mojtaba*, Gorgani Soghraa \\ Chemical Engineering Department, Faculty of Engineering, Razi University, Kermanshah, Iran \\ *Corresponding author: m_ahmadi@razi.ac.ir
}

Copyright (C) 2014 Horizon Research publishing all rights reserved.

\begin{abstract}
Fenton advanced oxidation processes (AOPs) have been examined for their effectiveness at degrading sodium dodecylbenzene sulfonate (SDBS).Central composite design (CCD) of experiments was used to study the effect of process parameters on oxidation of pollutant compounds. The concentrations of SDBS and $\mathrm{H}_{2} \mathrm{O}_{2}$ and $\mathrm{pH}$ were the independent variables. Experiments were performed at initial concentrations of SDBS 0.5, 1.5 and2.5 $\mathrm{mM}$, initial concentrations of $\mathrm{H}_{2} \mathrm{O}_{2} 0.5,1.5$ and $2.5 \mathrm{mM}$ and $\mathrm{pH}$ of 2, 3 and 4. A regression model was used to assess the influence of these independent variables on chemical oxygen demand (COD) removal. Analysis of variance (ANOVA) of the data and the regression model showed that $\mathrm{H}_{2} \mathrm{O}_{2}$ and $\mathrm{UV}$ irradiation had significant effects on COD removal.COD of the water decreased significantly with increasing the concentration of hydrogen peroxide. The negative coefficient of this variable $(\mathrm{pH})$ indicated that level of the COD removal decreased as the $\mathrm{pH}$ increased from 2 to 4 . Quadratic models were predicted for the response variable, i.e. COD removal, and the maximum model-predicted removals was38.48\%. Optimum conditions for this wastewater treatment was obtained based on the performance of the Fenton's oxidation in the experiment where the initial SDBS concentration was $1.68 \mathrm{mM}$ and the $\mathrm{H}_{2} \mathrm{O}_{2}$ initial concentration and $\mathrm{pH}$ were $2.27 \mathrm{mM}$ and 3.77 , respectively.
\end{abstract}

Keywords Advanced Oxidation, Fenton, Sodium Dodecylbenzene Sulfonate, Response Surface Methodology, Central Composite Design

\section{Introduction}

Linear alkylbenzene sulfonates (LAS) was introduced in the early sixties as the readily biodegradable replacement for the poorly biodegradable highly branched tetrapropylbenzene sulfonate. LAS contain an aromatic ring sulfonated at the para position and attached to a linear alkyl chain at any position except the terminal ones. The commercial product mainly consists of a complex mixture of various homologues and isomers, representing different alkyl chain lengths (ranging from 10 to 14 carbon atoms) and aromatic ring positions along the linear alkyl chain[1]. LAS production in the USA, Western Europe and Japan was $840 \times 10^{3}$ (metric) tons year ${ }^{-1}$ in 1994 . LAS represent more than $40 \%$ of all surfactants used; special attention has been paid to its occurrence and fate in the environment [2].

LAS are generally regarded as biodegradable surfactants but removal of them from wastewater has generally been restricted to conventional biological treatments. Their presence in municipal wastewater is variable depending on their use in industrial processing in addition to domestic activities. An average LAS concentration of $1-10 \mathrm{mg}^{-1}$ can be found in municipal wastewater treatment dealing only domestic wastewater [3] but this range is noticeably increased when industrial wastes from washing processes are also treated[4]. LAS biodegradation under aerobic conditions has been the main object of many studies and very high levels of biodegradation (97-99\%) have been found in some wastewater treatment plants using aerobic processes [2]. However, at LAS concentrations between 20 and $50 \mathrm{mg} \mathrm{l}^{-1}$ biodegradation may be restricted and at higher concentration of it, it can be even inhibited [5]. In light of this, exploring the potential of less conventional techniques to aid biological processes is of great interest. Various forms of AOPs have been investigated for treating wastewaters containing refractory compounds. These include the use of ozonation [6], $\mathrm{TiO}_{2}$ photocatalysis[7], Fenton oxidation [8], wet air oxidation[5, 9, 10] and electrochemical treatment[11].

Advanced oxidation processes (AOPs) are promising technologies that offer viable alternatives [12]. One available technique in the area of advanced oxidation process is based on the Fenton's reagent capabilities in production of hydroxyl radicals. Fenton's reagent, a homogeneous catalytic system comprising hydrogen peroxide and a ferrous 
salt, is another common AOP applied to the decomposition various organic pollutants in wastewater. The process relies on the fact that iron-catalyzed hydrogen peroxide decomposition leads to the formation of ${ }^{\bullet} \mathrm{OH}$ and other reactive free radicals, which are capable of oxidizing many organic pollutants and converting them to lower molecular weight compounds and eventually to carbon dioxide and water. Many metals have special oxygen transfer properties which improve the usage of hydrogen peroxide in treating wastewaters. Ferrous salts catalyze the hydrogen peroxide degradation, which yields and (Eq.(1)). The generated Fe (III) can be reduced by reaction with the exceeding $\mathrm{H}_{2} \mathrm{O}_{2}$ to form again ferrous ion in a catalytic mechanism (Eqs.(2) and (3)). Hydroxyl free radical is one of the most reactive chemical species known with an extremely high oxidation potential, $\mathrm{OH} / \mathrm{H}_{2} \mathrm{O}_{2}=+2.73 \mathrm{~V}$ [10]. Organic substances are oxidative and no selectively degraded by the generated hydroxyl radicals. The ferrous iron initiates the decomposition of hydrogen peroxide, resulting in the generation of hydroxyl radicals. The generation of the radicals involves a complex reaction sequence in an aqueous solution

$$
\begin{aligned}
\mathrm{Fe}^{2+}+\mathrm{H}_{2} \mathrm{O}_{2} & \rightarrow \mathrm{Fe}^{+}+\mathrm{HO}^{\cdot}+\mathrm{HO}^{-} \\
\mathrm{Fe}^{3+}+\mathrm{H}_{2} \mathrm{O}_{2} & \rightarrow \mathrm{HO}_{2}^{\cdot}+\mathrm{Fe}^{2+}+\mathrm{H}^{+} \\
\mathrm{Fe}^{3+}+\mathrm{HO}_{2}^{\cdot} & \rightarrow \mathrm{Fe}^{2+}+\mathrm{O}_{2}+\mathrm{H}^{+}
\end{aligned}
$$

Hydroxyl radicals can oxidize organics (RH) by abstraction of protons producing organic radicals (R.), which are highly reactive and can be further oxidized[13]

$$
\mathrm{RH}+\mathrm{OH}^{*} \rightarrow \mathrm{H}_{2} \mathrm{O}+\mathrm{R}^{\cdot} \rightarrow \text { furtheroxidation }
$$

Zhang et al. studied decomposition of the sodium dodecyl benzene sulfonate in aqueous titanium dioxide dispersions under highly concentrated solar light [14]. Ultrasonic irradiation for the removal of sodium dodecyl benzene suffocate (SDBS) from aqueous solutions has been investigated by Manousaki [15]. This study revealed that addition of $\mathrm{Fe}^{2+}$ either alone or in conjunction with $\mathrm{H} 2 \mathrm{O} 2$ (Fenton reagent) had a positive effect on degradation. The removal of sodium dodecyl benzene suffocate (SDBS), using silica/titania nanorods/nanotubes composite membrane with photocatalytic capability was carried out by Zhang [16]. The results showed that the removal of SDBS achieved $89 \%$ after $100 \mathrm{~min}$ by combining the photo catalysis with membrane filtration techniques. Méndez-Díaz examined the removal of the model surfactant sodium dodecylbenzene sulfonate (SDBS) from waters using several radical-based water treatment processes [17].

In the present work, Fenton AOPs have been examined for their effectiveness at degrading SDBS. Central composite design (CCD) of experiments was used to develop mathematical model and study the effect of process parameters on oxidation of pollutant compounds. The empirical model developed was used to determine the optimum concentrations of chemical reagents required for the effective treatment of SDBS.

\section{Materials and Methods}

\subsection{Experiment Set-up}

Fenton's oxidation experiments were carried out batch wise by placing $500 \mathrm{ml}$ Erlenmeyer flask as a reactor having the appropriate aqueous solution of SDBS solution in a shaking incubator $\left(\mathrm{T}: 25^{\circ} \mathrm{C}\right.$; agitation: $130 \mathrm{rpm}$ and time of the reaction:2 hrs). Several experiments were carried out according to the design of the work (see below for the details). During the experiments samples were taken from the vessel at the end of each experiment for analysis with respect to chemical oxygen demand (COD). Table 1 shows the arrangements of independent (regressors) and dependent variables used in the statistical analysis of the present study.

Table 1. Independent variables and their levels for central composite design used in the present study

\begin{tabular}{ccccccc}
\hline $\begin{array}{c}\text { Experim } \\
\text { ent no. }\end{array}$ & $\mathrm{A}$ & $\mathrm{B}$ & $\mathrm{C}$ & $\begin{array}{c}{[\mathrm{SDBS}]_{0}} \\
\mathrm{mM}\end{array}$ & $\begin{array}{c}{\left[\mathrm{H}_{2} \mathrm{O}_{2}\right]_{0}} \\
\mathrm{mM}\end{array}$ & $\mathrm{pH}$ \\
\hline 1 & -1 & -1 & -1 & 0.5 & 0.5 & 2 \\
2 & +1 & -1 & -1 & 2.5 & 0.5 & 2 \\
3 & -1 & +1 & -1 & 0.5 & 2.5 & 2 \\
4 & +1 & +1 & -1 & 2.5 & 2.5 & 2 \\
5 & -1 & -1 & +1 & 0.5 & 0.5 & 4 \\
6 & +1 & -1 & +1 & 2.5 & 0.5 & 4 \\
7 & -1 & +1 & +1 & 0.5 & 2.5 & 4 \\
8 & +1 & +1 & +1 & 2.5 & 2.5 & 4 \\
9 & -1 & 0 & 0 & 0.5 & 1.5 & 3 \\
10 & +1 & 0 & 0 & 2.5 & 1.5 & 3 \\
11 & 0 & -1 & 0 & 1.5 & 0.5 & 3 \\
12 & 0 & +1 & 0 & 1.5 & 2.5 & 3 \\
13 & 0 & 0 & -1 & 1.5 & 1.5 & 2 \\
14 & 0 & 0 & +1 & 1.5 & 1.5 & 4 \\
15 & 0 & 0 & 0 & 1.5 & 1.5 & 3 \\
16 & 0 & 0 & 0 & 1.5 & 1.5 & 3 \\
\hline & & & & & & \\
\hline
\end{tabular}

\subsection{Experimental Procedure}

Each experiment was carried out by adding sodium dodecyl benzene sulfonate (SDBS)(Aldrich Canada) to the reactor at the desired concentration. Hydrogen peroxide $\left(\mathrm{H}_{2} \mathrm{O}_{2}, 33 \% \mathrm{w} / \mathrm{v}\right.$, Fischer Scientific) and ferrous sulfate heptahydrate $\left(\mathrm{FeSO}_{4} .7 \mathrm{H}_{2} \mathrm{O}\right.$, Fluka) were added to the reactor at the beginning of the reaction. The $\mathrm{pH}$ was adjusted to 3.5 using hydrochloric acid. All the experiments were carried out with peroxide concentrations between 10 and $50 \mathrm{mg} / \mathrm{L}$ and $\mathrm{Fe}^{2+}$ concentrations of 1 to $5 \mathrm{mg} / \mathrm{L}$. Water samples were collected at different time intervals and were analyzed for chemical oxygen demand (COD) and peroxide concentration.Chemical oxygen demand (COD), in the wastewater samples was determined according to standard methods for the examination of water and wastewater [18]. 


\subsection{Experimental Design and Data Analysis}

A central composite design (CCD) in the form of factorial design was used for determining the effects of SDBS and hydrogen peroxide concentrations as well as $\mathrm{pH}$. Table 1 shows the arrangements of independent (regressors) and dependent variables used in the statistical analysis of the present study. This setup allowed the development of empirical equations as a function of initial concentration of SDBSand hydrogen peroxide (A), (B) respectively also $\mathrm{pH}$ (C). There were three first-order effects (terms in A, B and $\mathrm{C})$, three interaction effects (terms in $\mathrm{AB}, \mathrm{AC}$ and $\mathrm{BC}$ ), and tree second-order effects $\left(\mathrm{A}^{2}, \mathrm{~B}^{2}\right.$ and $\left.\mathrm{C}^{2}\right)$, according to the generalized equation (polynomial multiple regression equation) $[19,20]$ :

$$
\begin{aligned}
Y=\beta_{0}+\beta_{1} A & +\beta_{2} B+\beta_{3} C+\beta_{1} A^{2}+\beta_{2} B^{2}+\beta_{3} C^{2} \\
& +\beta_{1} A_{2}+B \beta_{1} A_{3}+C \beta_{2} B_{3}
\end{aligned}
$$

The predicted response $(\mathrm{y})$ was correlated therefore to the set of regression coefficients (10 coefficients): the linear ( $\beta_{1}$, $\left.\beta_{2}, \beta_{3}\right)$, cross products $\left(\beta_{12}, \beta_{13}\right.$ and $\left.\beta_{23}\right)$ and quadratic $\left(\beta_{11}, \beta_{22}, \beta_{33}\right) . C C D$ was developed using the Design Expert software (Version 6.0.6, Stat-Ease Inc., MN, USA). Each factor is varied over three levels: the high level $(+1)$, the low level $(-1)$, the center points (coded level 0 ).

\section{Results and Discussion}

In the present work the relationships between COD removal of the treated solution and three controllable factors (concentrations of SDBS and $\mathrm{H}_{2} \mathrm{O}_{2}$ and $\mathrm{pH}$ ) were investigated. The results obtained from applying $\mathrm{CCD}$ as described earlier, were examined by the analysis of variance (ANOVA) to assess the "goodness of fit", Only terms, i.e. factors, and the related interactions found statistically significant were included in the model. For the COD removal model, $\beta_{23}$ was non-significant and dropped. A new ANOVA was then performed for the reduced model. The model for the COD removals was significant by the F-test at $5 \%$ significance level (Prob $<0.05$ ). The following fitted regression models (equations in terms of coded values for the predictors) were used to quantitatively describe the effects of initial concentration of SDBS (A) and $\mathrm{H}_{2} \mathrm{O}_{2}$ (B) as well as $\mathrm{pH}$ (C) (the result of ANOVA is shown on Table 2):

$$
\begin{gathered}
\mathrm{Y}=33.72+1.35 \mathrm{~A}+2.41 \mathrm{~B}-0.95 \mathrm{C}+3.99 \mathrm{AB}+4.19 \mathrm{AC}+5.92 \mathrm{BC}- \\
16.26 \mathrm{~A} 2+2.58 \mathrm{~B} 2-3.87 \mathrm{C} 2
\end{gathered}
$$

The experimental data showed a good fit with the polynomial equations, which were statistically significant at $\mathrm{P}<0.05$ levels. The coefficients of determination, $\mathrm{R}^{2}$ and adjust $\mathrm{R}^{2}$, for COD reduction were 0.99 and 0.98 , respectively. Three dimensional response surface plot for COD removal in Fenton response surfaces were used to show the changes for the COD reduction as a function of initial concentration of SDBS and pH (Figures 1, 2 and 3). The actual values obtained from experimental studies was compared to the estimated values from regression models (Table 3).Three dimensional response surface plots were made with the vertical axes showing COD reduction, and each of the two horizontal axes representing any two of the three independent variables.

Table 2. ANOVA of the fitted models for the COD removal

\begin{tabular}{cccccc}
\hline Source & SS & Df & MS & F-value $^{*}$ & p-value \\
\hline Model & 1745.75 & 9 & 193.97 & 93.19 & $<0.0001$ \\
$\mathrm{~A}$ & 18.11 & 1 & 18.11 & 8.70 & 0.0256 \\
$\mathrm{~B}$ & 58.24 & 1 & 58.24 & 27.98 & 0.0018 \\
$\mathrm{C}$ & 9.11 & 1 & 9.11 & 4.38 & 0.0814 \\
$\mathrm{AB}$ & 127.39 & 1 & 127.39 & 61.20 & 0.0002 \\
$\mathrm{AC}$ & 140.76 & 1 & 140.76 & 67.63 & 0.0002 \\
$\mathrm{BC}$ & 280.76 & 1 & 280.76 & 134.89 & $<0.0001$ \\
$\mathrm{~A}^{2}$ & 697.27 & 1 & 697.27 & 334.99 & $<0.0001$ \\
$\mathrm{~B}^{2}$ & 17.51 & 1 & 17.51 & 8.41 & 0.0273 \\
$\mathrm{C}^{2}$ & 39.44 & 1 & 39.44 & 18.95 & 0.0048 \\
Residual & 12.49 & 6 & 2.08 & & \\
Total & 1758.24 & 15 & & & \\
\hline
\end{tabular}

*Significance at the $5 \%$ level.

Table 3. Experimental and theoretically predicted values for COD removal in the present study

\begin{tabular}{ccc}
\hline \multirow{2}{*}{ Experiment no. } & \multicolumn{2}{c}{ COD removal (\%) } \\
& Actual value & Predicted value \\
\hline 1 & 27.47 & 27.47 \\
3 & 14.66 & 13.79 \\
4 & 13.50 & 12.46 \\
5 & 14.52 & 14.75 \\
6 & 5.90 & 5.32 \\
7 & 7.74 & 8.42 \\
8 & 13.50 & 14.01 \\
9 & 33.43 & 33.08 \\
10 & 15.01 & 16.11 \\
11 & 18.47 & 18.80 \\
12 & 33.10 & 33.88 \\
13 & 38.05 & 38.71 \\
14 & 29.11 & 30.80 \\
15 & 29.15 & 28.89 \\
16 & 35.10 & 33.72 \\
\hline
\end{tabular}

Figure 1 shows the dependence of COD removal on SDBS concentration and $\mathrm{pH}$, when hydrogen peroxide concentration was at a fixed level (i.e., $B=2.5 \mathrm{mM}$ ). At a given SDBS concentration, increasing level of $\mathrm{pH}$ from its low level to the midlevel, the COD removal increases thereafter it starts to decrease, although the extent of the decreasing level is somewhat different for each level of the 
$\mathrm{pH}$, greatest level of the COD removal corresponds to the midlevel SDBS concentration when $\mathrm{pH}$ was at its midlevel level.
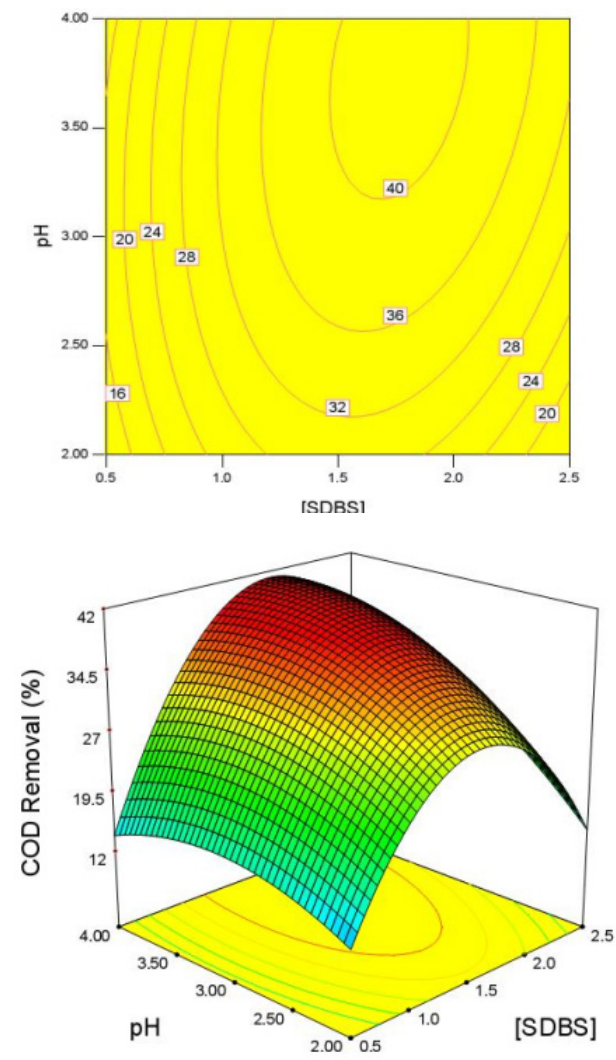

Figure 1. Response surface plot and contour plot of COD removal as a function of initial concentration of SDBS and $\mathrm{pH},\left(\left[\mathrm{H}_{2} \mathrm{O}_{2}\right]_{0}=2.5 \mathrm{mM}\right)$

Figure 2 shows the dependency COD reduction on $\mathrm{H} 2 \mathrm{O} 2$ concentration and $\mathrm{pH}$ when SDBS concentration was kept at its high level $(\mathrm{A}=+1)$. COD removal increases as hydrogen peroxide concentration increased to its high level as high level of $\mathrm{pH}$.

Figure 3 shows the dependence of COD removal on both

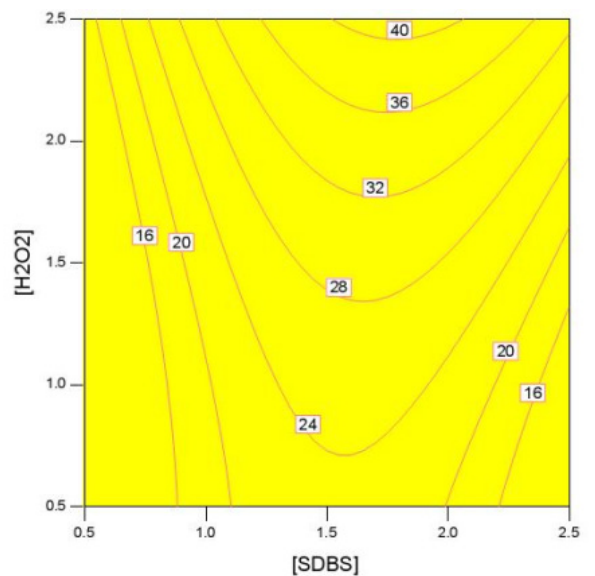

the SDBS concentration and hydrogen peroxide concentration, setting at its central level $(\mathrm{C}=+1)$. The plot shows a strong degree of curvature of three dimensional surfaces. COD removal increases as hydrogen peroxide concentration increased to its high level and at a given $\mathrm{H} 2 \mathrm{O} 2$ concentration, increasing level of SDBS concentration from its low level to the midlevel, the COD removal increases thereafter it starts to decrease.
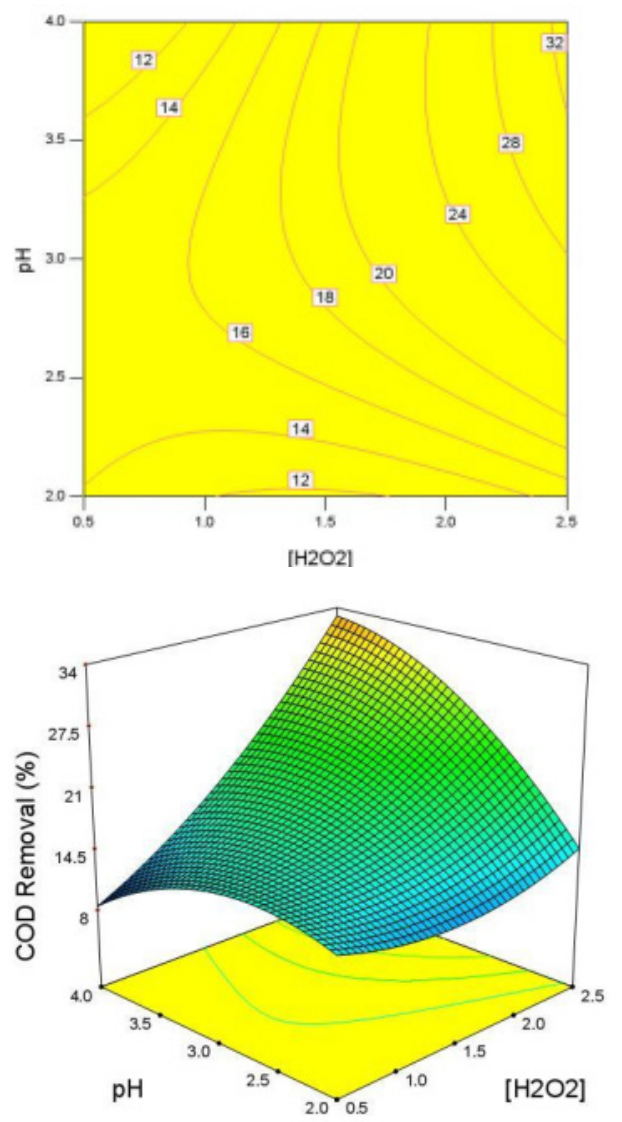

Figure 2. Response surface plot and Plot of COD removal as a function of initial concentration of hydrogen peroxide and $\mathrm{pH}([\mathrm{SDBS}] 0=2.5 \mathrm{mM})$

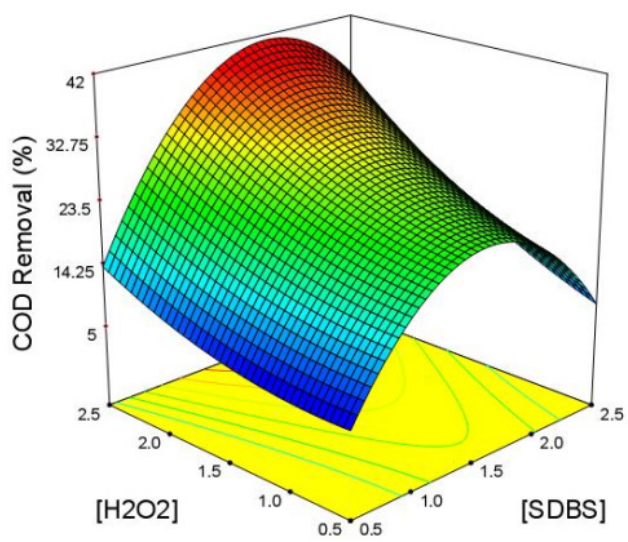

Figure 3. Response surface plot and Contour plot of $\mathrm{COD}$ removal as a function of initial concentration of hydrogen peroxide and SDBS $(\mathrm{pH}=4)$ 


\section{Conclusions}

Fenton's oxidation for the synthetic wastewater containing SDBS treatment was studied using central composite design and response surface methodology. The optimum conditions for this treatment was achieved by setting the experiment with hydrogen peroxide concentration at $2.5 \mathrm{mM}$ (high level) while the other two regressor variables, i.e., SDBS concentration and $\mathrm{pH}$ level were at $1.5 \mathrm{mM}$ and 3 , respectively. Initial concentration of hydrogen peroxide was found to be more important for COD removal. Degradation capacity of the Fenton's reagent was less pronounced in the range studied. Nonselective oxidizing role of the hydroxyl radicals toward aromatics during initial stages of the aromatic ring sulfonated removal may change as the reaction proceeds and other oxidants appear. It was possible therefore, to obtain the empirical models to describe and predict removal of the major pollutants. Adjustment of the quadratic model with the experimental data was satisfactory. Analysis of variance showed a high coefficient of determination value 0.99. It was possible therefore, to develop the empirical equations describing and predicting the removal of the pollutants.

\section{REFERENCES}

[1] A. Di Corcia, et al., Fate of Linear Alkyl Benzenesulfonates, Coproducts, and Their Metabolites in Sewage Treatment Plants and in Receiving River Waters, Environmental Science \& Technology 33(22) (1999) 4119-4125.

[2] M.J. Scott and M.N. Jones, The biodegradation of surfactants in the environment, Biochimica et BiophysicaActa (BBA) Biomembranes 1508(1-2) (2000) 235-251.

[3] F. J.A., et al., Fate of secondary alkane sulfonate surfactants during municipal wastewater treatment, Water Res. 29(5) (1995) 1301-1307.

[4] F.J. Beltrán, J.F. García-Araya, and P.M. Álvarez, Sodium Dodecylbenzenesulfonate Removal from Water and Wastewater. 1. Kinetics of Decomposition by Ozonation, Industrial \& Engineering Chemistry Research 39(7) (2000) 2214-2220.

[5] D.A. Patterson, et al., Wet Air Oxidation of Linear Alkylbenzene Sulfonate 1. Effect of Temperature and Pressure, Industrial \& Engineering Chemistry Research 40(23) (2001) 5507-5516.

[6] B. Delanghe, C.I. Mekras, and N.J.D. Graham, Aqueous Ozonation Of Surfactants: A Review, Ozone: Science \& Engineering 13(6) (1991) 639-673.
[7] T. S. and I. T., Effects of photolytic pretreatment on biodegradation and detoxification of surfactants in anaerobic digestion, Water Sci. Technol 28(7) (1993) 103-110.

[8] S.H. Lin, c.M. Lin, and H.G. Leu, Operating characteristics and kinetic studies of surfactant wastewater treatment by Fenton oxidation, Water Research 33(7) (1999) 1735-1741.

[9] D.A. Patterson, et al., Biodegradability of linear alkylbenzene sulfonates subjected to wet air oxidation, J. Chem. Technol. Biotechnol. 77(9) (2002) 1039-1049.

[10] D. Mantzavinos, et al., Chemical treatment of an anionic surfactant wastewater: electrospray-ms studies of intermediates and effect on aerobic biodegradability, Water Research 35(14) (2001) 3337-3344.

[11] S. Zor, et al., The electrochemical degradation of linearalkylbenzenesulfonate (LAS) on platinum electrode, Water Research 32(3) (1998) 579-586.

[12] L. O., O. E., and B. A.M., Photochemical processes for water treatment, Chem. Rev. 93 (1993) 671-675.

[13] L. S.H. and L. C.C., Fenton process for treatment of desizing wastewater. 318 (1997), pp. 2050-2056, Water Res. 31(8) (1997) 2050-2056.

[14] T. Zhang, et al., Photocatalytic decomposition of the sodium dodecylbenzene sulfonate surfactant in aqueous titania suspensions exposed to highly concentrated solar radiation and effects of additives, Applied Catalysis B: Environmental 42(1) (2003) 13-24.

[15] E. Manousaki, et al., Degradation of sodium dodecylbenzene sulfonate in water by ultrasonic irradiation, Water Research 38(17) (2004) 3751-3759.

[16] H. Zhang, et al., The removal of sodium dodecylbenzene sulfonate surfactant from water using silica/titaniananorods/nanotubes composite membrane with photocatalytic capability, Applied Surface Science 252(24) (2006) 8598-8604.

[17] J. Méndez-Díaz, et al., Advanced oxidation of the surfactant SDBS by means of hydroxyl and sulphate radicals, Chemical Engineering Journal 163(3) (2010) 300-306.

[18] S.C. Lenore, E.G. Arnold, and D.E. Andrew, Standard Methods for the Examination of Water and Wastewater Washington, D.C, USA, American Public Health Association 1999

[19] G.G. Vining, Statistical Methods for Engineers. Duxburg Press, An International Thomason Publishing, London 1V7AA 2003

[20] L.L. Lapin, Modern Engineering Statistics. 1997, Wards worth Publishing Company: Belmont, CA, USA. 